\title{
Towards limiting QT interval prolongation and arrhythmia risk in citalopram use
}

\author{
Yihong Zhang ${ }^{1}$, Adrian Baranchuk ${ }^{2}$, Jules C. Hancox ${ }^{1}$ \\ ${ }^{1}$ School of Physiology and Pharmacology and Cardiovascular Research Laboratories, \\ Medical Sciences Building, University of Bristol, Bristol, United Kingdom \\ ${ }^{2}$ Department of Cardiology, Kingston General Hospital, Queens University, Kingston, Ontario, Canada
}

\section{Psychotropic actions of citalopram and escitalopram}

Citalopram is a serotonin-selective reuptake inhibitor (SSRI) widely used in the treatment of major depression and sometimes also anxiety-associated conditions, obsessive compulsive disorder and behavior disturbances associated with dementia [1,2]. Citalopram is relatively well tolerated and has been considered to have comparatively low potential for drug-drug interactions and anti-adrenergic and anti-cholinergic effects, making it an attractive treatment option for elderly patients [1,2]. The drug is chiral and the serotonin reuptake inhibitory activity of citalopram resides in the $\mathrm{S}(+)$-enantiomer (available as escitalopram) with no therapeutic benefit of the $\mathrm{R}(-)$-enantiomer. There is limited evidence that at equivalent doses (i.e. matched concentrations of the S-enantiomer), escitalopram is more effective clinically than is citalopram, raising the possibility of a potential inhibitory effect of the R-enantiomer [3-5]. Data from studies, in which serotonin reuptake transporter (SERT) occupancy has been examined in humans, suggest that on repeated dosing with racemic citalopram, R-citalopram levels may exceed those of the S-enantiomer, leading to reduced S-citalopram occupancy of SERT [6].

\section{Citalopram, escitalopram and QT prolongation}

A number of psychotropic drugs are associated with prolongation of rate-corrected $\mathrm{QT}\left(\mathrm{QT}_{\mathrm{c}}\right)$ interval and the associated risk of torsades de pointes (TdP) arrhythmia (see: www.crediblemeds.org and $[7,8])$. Citalopram has been reputed to have a good safety profile within the clinical concentration range making it an attractive alternative to tricyclic antidepressants [2, 9]. However, there is some evidence of QT prolongation and arrhythmia with citalopram (for reviews see [7, 8, 10]). Reviews of data from United States and Swedish adverse effect registries note $\sim 20$ cases of $\mathrm{TdP}$ associated with citalopram $[8,11]$, with a number of additional case reports in the literature (for a recent review see: [12]). Some case reports of QT prolongation have also now emerged for escitalopram (e.g. [13-16]).

Potential dangers with normal use of citalopram came to prominence in safety updates from the United States Food and Drug Administration (FDA) $[17,18]$ as well as from other regulatory agencies including the European Medicines Agency (EMA) [19] and United Kingdom Medicines and Healthcare Products Regulatory Agency (MHRA) [20]. In August of 2011 [17] and in a subsequent update in March 2012 [18], the FDA produced revised recommendations and warnings for citalopram, limiting the maximum daily dose for all patients and reducing this further for older patients. The safety advisory information resulted from evaluation of 'thorough QT' data from randomized, double-blind, placebo-controlled cross-over human subject studies on citalopram and escitalopram. Dose-dependent $\mathrm{QT}_{\mathrm{c}}$ prolongation was found for both drugs in healthy volunteers, with greater effects for citalopram than therapeutically equivalent concentrations of escitalopram. The extent of $\mathrm{QT}_{\mathrm{c}}$ prolongation seen with given doses of citalopram compared to therapeutically equivalent escitalopram doses was suggestive of cardiac

Address for correspondence: Jules C. Hancox, PhD, FSB. FBPharmacolS, School of Physiology and Pharmacology and Cardiovascular Research Laboratories, Medical Sciences Building, University of Bristol, Bristol, BS8 1TD, United Kingdom, e-mail: jules.hancox@bristol.ac.uk

Received: 31.05.2014 Accepted: 21.07.2014 
actions of both citalopram enantiomers. Indeed, the FDA safety announcement included a speculation that whilst antidepressant effects of citalopram are limited to the S-enantiomer, the difference between the two forms of drug on the $\mathrm{QT}_{\mathrm{c}}$ interval "presumably means that the QT effects are not limited to the $S$-isomer" $[18,21]$.

\section{Citalopram and hERG channel inhibition}

Virtually all drugs associated with $\mathrm{QT}_{\mathrm{c}}$ interval share in common the ability to produce pharmacological inhibition of ion channels mediating the cardiac rapid delayed rectifier potassium current ("I $\mathrm{Kr}_{\mathrm{Kr}}$ ) and its cloned equivalent hERG - humanEther-à-go-go-Related Gene [22]. $\mathrm{I}_{\mathrm{Kr}}$ regulates ventricular action potential duration and drugs that inhibit $\mathrm{I}_{\mathrm{Kr}} / \mathrm{hERG}$ consequently delay ventricular repolarization and thereby prolong the $\mathrm{QT}_{\mathrm{c}}$ interval [22]. Citalopram was first reported to inhibit the hERG potassium channel in 2002 in a study from our laboratory, with a half maximal inhibitory concentration $\left(\mathrm{IC}_{50}\right)$ for hERG current $\left(\mathrm{I}_{\mathrm{hERG}}\right)$ of $3.97 \mu \mathrm{M}$, slightly less than that for another SSRI, fluoxetine, in the same study $(1.5 \mu \mathrm{M})$ [23]. A subsequent independent study confirmed hERG block by citalopram (with a lower $\mathrm{IC}_{50}$ of $0.95 \mu \mathrm{M}$ [24]). Therapeutic benefits of citalopram for major depression appear to require plasma levels of 50 $\mathrm{ng} / \mathrm{mL}$ or greater [25], with maximal levels of $\sim 120$ $\mathrm{ng} / \mathrm{mL}$ at therapeutic doses [9]. With a caveat that citalopram is highly lipophilic (so that the possibility of tissue accumulation must be borne in mind), therapeutic total plasma levels are expected to be below $\sim 200-300 \mathrm{nM}$, though raised levels could feasibly be attained in overdose or with metabolic impairment. hERG block at normal concentrations may therefore normally not be high, but could be increased in situations of impaired metabolism, drug overdose or be exacerbated by cardiac effects of electrolyte abnormalities.

Given that the R-enantiomer of citalopram is without therapeutic benefit and, consequently, that for the racemate twice the total quantity of citalopram is present than for escitalopram for a given therapeutically effective dose of the S-enantiomer, it has been suggested that moving patients from citalopram to escitalopram may be prudent in terms of reducing cardiac risk [26]. Until recently, however, there has been little or no information as to whether or not the two citalopram enantiomers are similar in their ability to inhibit hERG. Very recently, however, Chae et al. [27] have reported an in vitro comparison of the hERG-blocking propensity of citalopram and escitalopram, with approximately similar $\mathrm{IC}_{50}$ values for citalopram and of escitalopram. The R-enantiomer was not directly investigated in that study [27]. We have compared $\mathrm{S}(+), \mathrm{R}(-)$ citalopram enantiomers and the racemic mixture on hERG channels using patch clamp recording at physiological temperature to elicit $\mathrm{I}_{\mathrm{hERG}}(c f$ [28]). As shown in Figures $1 \mathrm{~A}-\mathrm{C}$, equal concentrations of citalopram, escitalopram and R-citalopram produced similar levels of $\mathrm{I}_{\mathrm{hERG}}$ inhibition and concentration response relations (shown in Fig. 1D) yielded similar $\mathrm{IC}_{50}$ values (of $0.68 \pm 0.08 \mu \mathrm{M}$, $0.70 \pm 0.06 \mu \mathrm{M}$ and $0.67 \pm 0.02 \mu \mathrm{M})$ for citalopram, escitalopram and R-citalopram respectively, under our recording conditions. These results complement and extend the recent findings of Chae et al. [27] and demonstrate clearly that, whilst only the S-citalopram enantiomer is of psychotropic value, both $\mathrm{S}(+)$ and $\mathrm{R}(-)$ citalopram enantiomers can be expected to act on $\mathrm{I}_{\mathrm{Kr}} / \mathrm{hERG}$ acutely and, thereby, to delay repolarization.

\section{Limiting cardiac risk}

A logical conclusion from the information now available on hERG-blocking actions of citalopram enantiomers together with the thorough QT data reported by the FDA [18] is that escitalopram should confer a lower cardiac risk than citalopram for therapeutically equivalent concentrations and therefore should be used clinically in place of racemic citalopram. Whilst our own findings (Fig. 1) support such a notion, some qualification is prudent at this time. First, it is known that additional concomitant ion channel blocking effects can mitigate consequences of hERG channel inhibition and citalopram has been found to exert an additional weak inhibition of cardiac L-type Ca current $\left(\mathrm{I}_{\mathrm{Ca}, \mathrm{L}}\right)$ and to modify $\mathrm{I}_{\mathrm{Ca}, \mathrm{L}}$ kinetics $[23$, $24,29]$. It is feasible that this weak action on $\mathrm{I}_{\mathrm{Ca}, \mathrm{L}}$ may mitigate effects of hERG blockade at high citalopram concentrations. At present it is not yet fully established how these two channel effects of citalopram interact and, indeed, whether the $\mathrm{I}_{\mathrm{Ca}, \mathrm{L}}$ action of citalopram is stereoselective. Additional information on these points would be helpful. Second, cardiac side effects of citalopram need not commence immediately following dosing/ingestion. One mechanism for delayed drug effects on the $\mathrm{QT}_{\mathrm{c}}$ interval is through inhibition of $\mathrm{hERG}$ channel trafficking to the cell membrane but, whilst there is some evidence that citalopram and escitalopram are in principle able to produce this effect [27], marked suppression of hERG function 


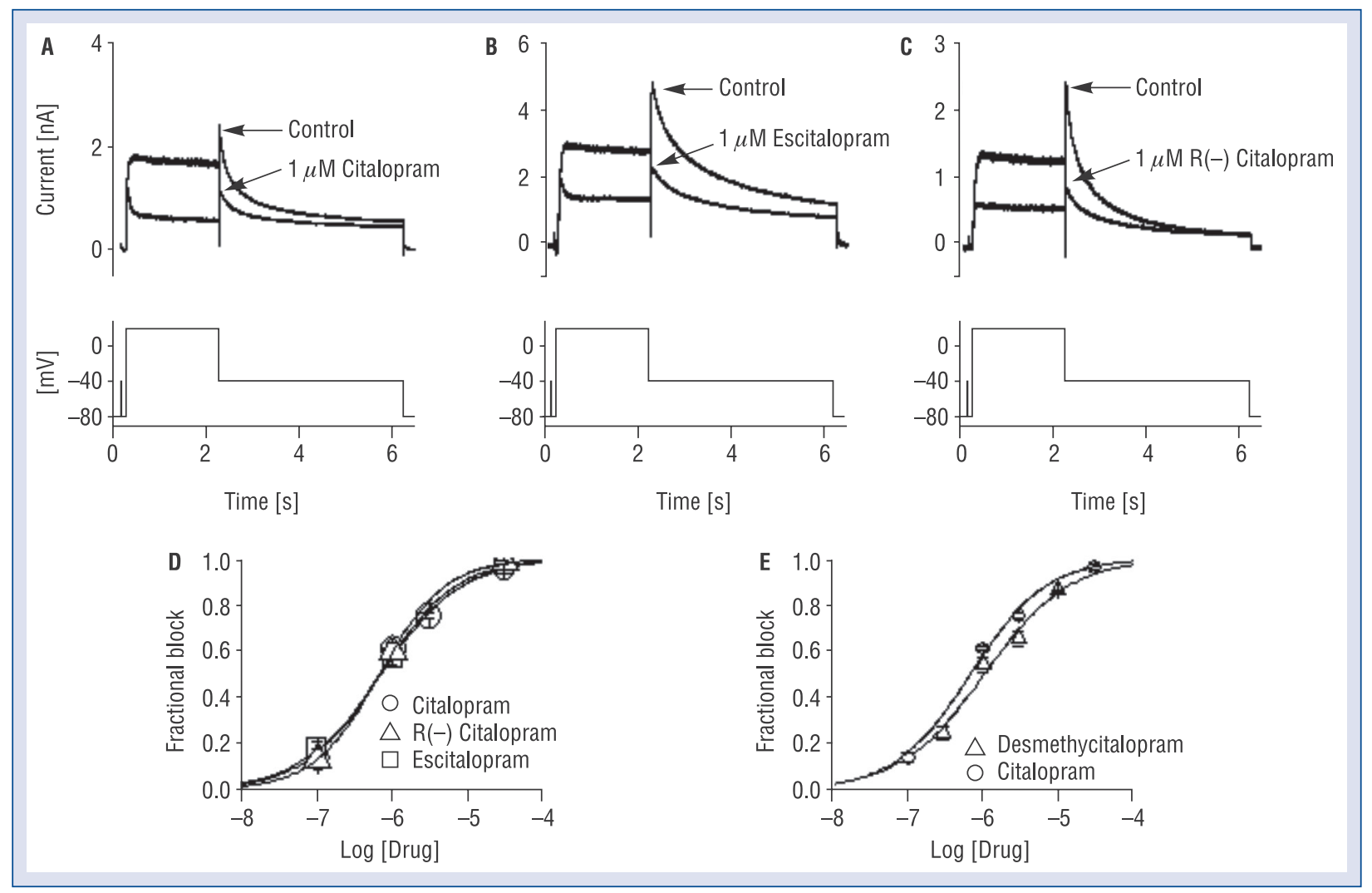

Figure 1. Comparative inhibition of hERG potassium channels by citalopram enantiomers; A-C. Upper traces show representative traces of $\mathrm{I}_{\mathrm{hERG}}$ elicited from HEK293 cells expressing hERG by the voltage clamp protocol shown in the lower panel, in control and after $1 \mu \mathrm{M}$ of citalopram (A), escitalopram (B), R(-) citalopram (C). D. Mean ( \pm SEM) concentration-response plots, overlain, for inhibition of $\mathrm{I}_{\mathrm{hERG}}$ (tail current) by citalopram, escitalopram and $\mathrm{R}(-)$ citalopram. $I C_{50}$ values are given in the main text. Hill-coefficient values for citalopram, escitalopram and $\mathrm{R}(-)$ citalopram were respectively: $0.86 \pm 0.09,0.81 \pm 0.06,0.99 \pm 0.03$; E. Mean $( \pm S E M)$ concentration-response plots, overlain, for citalopram and desmethycitalopram. $\mathrm{IC}_{50}$ values were, respectively, $0.68 \pm 0.08 \mu \mathrm{M}$ and $1.05 \pm 0.17 \mu \mathrm{M}$ (Hill co-efficients $0.86 \pm 0.09$ and $0.78 \pm 0.11) ; n \geq 5$ cells per data-point in $D$ and $E$.

through this mechanism appears likely to require comparatively high drug concentrations ([27] and Zhang and Hancox, unpublished). An alternative possibility is that delayed citalopram effects may involve accumulation not simply of citalopram itself but also of citalopram's main metabolite desmethylcitalopram [30]. We have obtained evidence that desmethylcitalopram can indeed inhibit hERG, albeit with slightly reduced potency compared to the parent compound (Fig. 1E). At this time we do not know how the parent drug and metabolite interact when hERG channels are exposed to both molecules simultaneously or whether the inhibitory effect of the metabolite is stereoselective. This information would be also useful to obtain. Third, a recent analysis of case reports of TdP with SSRI use highlighted female sex and hypokalemia as present in a number of
TdP cases with citalopram [12]. Whilst these are known risk factors in general for drug-induced TdP [31], specific mechanistic information would be valuable as to how they exacerbate cardiac actions of citalopram. However, whilst it is important for these qualifications to be stated, it nevertheless seems reasonable to agree with a view that R-citalopram may serve largely to contribute to unwanted cardiac effects of citalopram and, therefore, that the ability in clinical practice to replace citalopram with lower dose formulations of the S-enantiomer may be of significant value in respect of reducing cardiac risk [26].

Acknowledgement of Funding: Funding by the British Heart Foundation (PG/10/17 and $\mathrm{PG} / 12 / 69)$ is gratefully acknowledged.

Conflicts of interest: None declared 


\section{References}

1. Pollock BG. Citalopram: A comprehensive review. Expert Opin Pharmacother, 2001; 2: 681-698.

2. Keller MB. Citalopram therapy for depression: A review of 10 years of European experience and data from U.S. clinical trials. J Clin Psychiatry, 2000; 61: 896-908.

3. Storustovu S, Sanchez C, Porzgen P et al. R-citalopram functionally antagonises escitalopram in vivo and in vitro: evidence for kinetic interaction at the serotonin transporter. $\mathrm{Br} \mathrm{J}$ Pharmacol, 2004; 142: 172-180.

4. Sanchez C. The pharmacology of citalopram enantiomers: The antagonism by R-citalopram on the effect of S-citalopram. Basic Clin Pharmacol Toxicol, 2006; 99: 91-95.

5. Zhong H, Haddjeri N, Sanchez C. Escitalopram, an antidepressant with an allosteric effect at the serotonin transporter: A review of current understanding of its mechanism of action. Psychopharmacology (Berl), 2012; 219: 1-13.

6. Kasper S, Sacher J, Klein N et al. Differences in the dynamics of serotonin reuptake transporter occupancy may explain superior clinical efficacy of escitalopram versus citalopram. Int Clin Psychopharmacol, 2009; 24: 119-125.

7. Witchel HJ, Hancox JC, Nutt DJ. Psychotropic drugs, cardiac arrhythmia, and sudden death. J Clin Psychopharmacol, 2003; 23: 58-77.

8. Wenzel-Seifert K, Wittmann M, Haen E. QTc prolongation by psychotropic drugs and the risk of torsade de pointes. Dtsch Arztebl Int, 2011; 108: 687-693.

9. Parker NG, Brown CS. Citalopram in the treatment of depression. Ann Pharmacother, 2000; 34: 761-771.

10. Vieweg WV, Hasnain M, Howland RH et al. Citalopram, QTc interval prolongation, and torsade de pointes. How should we apply the recent FDA ruling? Am J Med, 2012; 125: 859-868.

11. Astrom-Lilja C, Odeberg JM, Ekman E, Hagg S. Drug-induced torsades de pointes: A review of the Swedish pharmacovigilance database. Pharmacoepidemiol Drug Saf, 2008; 17: 587-592.

12. Kogut C, Breden-Crouse E, Vieweg WV et al. Selective serotonin reuptake inhibitors and torsades de pointes. New concepts and new directions derived from systematic review of case reports. Ther Adv Drug Safe, 2013; 4: 189-198.

13. Tseng PT, Lee Y, Lin YE, Lin PY. Low-dose escitalopram for 2 days associated with corrected QT interval prolongation in a middle-aged woman: A case report and literature review. Gen Hosp Psychiatry, 2012; 34: 210-215.

14. Mohammed R, Norton J, Geraci SA, Newman DB, Koch CA. Prolonged QTc interval due to escitalopram overdose. J Miss State Med Assoc, 2010; 51: 350-353.

15. van Gorp F, Whyte IM, Isbister GK. Clinical and ECG effects of escitalopram overdose. Ann Emerg Med, 2009; 54: 404-408.

16. Baranchuk A, Simpson CS, Methot M, Gibson K, Strum D. Corrected QT interval prolongation after an overdose of escitalo- pram, morphine, oxycodone, zopiclone and benzodiazepines. Can J Cardiol, 2008; 24: e38-e40.

17. FDA Drug Safety Communication: Abnormal heart rhythms associated with high doses of Celexa (citalopram hydrobromide) http://www. fda.gov/Drugs/DrugSafety/ucm269086.htm (accessed May 23 2014).

18. FDA Drug Safety Communication: Revised recommendations for Celexa (citalopram hydrobromide) related to a potential risk of abnormal heart rhythms with high doses. http://www.fda.gov/ /drugs/drugsafety/ucm297391.htm (accessed May 23 2014).

19. Pharmacoviglence working party (PhVWP) November 2011. Escitalopram: Risk of QT interval prolongation. http://www. ema.europa.eu/docs/en_GB/document_library/Report/2011/11/ WC500117988.pdf (accessed May 23 2014).

20. Citalopram and escitalopram: QT interval prolongation: New maximum daily dose restrictions (including in elderly patients), contraindications, and warnings http://www.mhra.gov.uk/Safety-information/ /DrugSafetyUpdate/CON137769 (accessed May 23 2014).

21. Temple R, Laughren T, Stockbridge N. Removal from labeling of 60-mg citalopram dose. Pharmacoepidemiol Drug Saf, 2012; 21: 784-786.

22. Hancox JC, McPate MJ, El Harchi A, Zhang YH. The hERG potassium channel and hERG screening for drug-induced torsades de pointes. Pharmacology Therapeutics, 2008; 119: 118-132.

23. Witchel HJ, Pabbathi VK, Hofmann G, Paul AA, Hancox JC. Inhibitory actions of the selective serotonin re-uptake inhibitor citalopram on HERG and ventricular L-type calcium currents. FEBS Lett, 2002; 512: 59-66.

24. Fossa AA, Gorczyca W, Wisialowski T et al. Electrical alternans and hemodynamics in the anesthetized guinea pig can discriminate the cardiac safety of antidepressants. J Pharmacol Toxicol Methods, 2007; 55: 78-85.

25. Ostad HE, Tadic A, Wagner S et al. Association between citalopram serum levels and clinical improvement of patients with major depression. J Clin Psychopharmacol, 2011; 31: 281-286.

26. Keller DL. Prescribe escitalopram instead of citalopram. Am J Med, 2013; 126: e21.

27. Chae YJ, Jeon JH, Lee HJ et al. Escitalopram block of hERG potassium channels. Naunyn Schmiedebergs Arch Pharmacol, 2014; 387: 23-32.

28. Zhang YH, Cheng H, Alexeenko VA, Dempsey CE, Hancox JC. Characterization of recombinant $\mathrm{hERG} \mathrm{K}^{+}$channel inhibition by the active metabolite of amiodarone desethyl-amiodarone. J Electrocardiol, 2010; 43: 440-448.

29. Zahradnik I, Minarovic I, Zahradnikova A. Inhibition of the cardiac L-type calcium channel current by antidepressant drugs. J Pharmacol Exp Ther, 2008; 324: 977-984.

30. Tarabar AF, Hoffman RS, Nelson L. Citalopram overdose: Late presentation of torsades de pointes (TdP) with cardiac arrest. J Med Toxicol, 2008; 4: 101-105.

31. Viskin S, Justo D, Halkin A, Zeltser D. Long QT syndrome caused by noncardiac drugs. Prog Cardiovasc Dis, 2003; 45: 415-427. 